\title{
Cardiomiopatia de Takotsubo Recorrente: Um Enigma ainda não Resolvido
}

\author{
Recurrent Takotsubo Cardiomyopathy: A Puzzle Yet to be Solved
}

Kenan Yalta ${ }^{1 \oplus}$ e Caglar Kaya ${ }^{1}$

Trakya University, School of Medicine, Cardiology Department, ${ }^{1}$ Edime - Turquia

Nos últimos anos, a Cardiomiopatia de Takotsubo (CTT) ganhou um amplo reconhecimento como uma forma transitória de disfunção miocárdica, geralmente surgindo em resposta a certos fatores desencadeantes de estresse associados à descarga adrenérgica, e foi relatado em estudos anteriores que o mesmo tem taxas de recorrência variáveis. ${ }^{1-7} \mathrm{Em}$ sua revisão sistemática ${ }^{1}$ recentemente publicada, Campos et al. ${ }^{1}$ sugeriram certos fatores associados à recorrência da CTT, incluindo índice de massa corporal (IMC) mais baixo, gênero feminino, gradiente médio-ventricular (GMV) já existente e proximidade temporal com o evento índice (inicial) da CTT. Concordamos plenamente com esses fatores de risco específicos neste cenário. No entanto, gostaríamos de ter mais informações sobre sua análise e fazer alguns comentários no contexto geral de recorrência da CTT. Como descrito abaixo, acreditamos que existam duas categorias básicas de pacientes com CTT que são mais propensos a ter recorrências futuras na prática clínica:

- A primeira categoria compreende os casos de CTT associados a um gatilho puramente mecânico, incluindo o GMV. ${ }^{1}$ Essa forma de evolução da CTT foi encontrada principalmente no contexto de cardiomiopatia hipertrófica $(\mathrm{CMH})$ e doença cardíaca hipertensiva, geralmente com uma pequena cavidade ventricular (mesmo sem GMV evidente em repouso), e parece ser desencadeada por aumentos súbitos e excessivos no GMV, levando a um padrão de balonismo apical, 2,3,6 geralmente de forma recorrente. ${ }^{1}$ Portanto, a reconstrução cirúrgica do médioventrículo pode ser obrigatória em certos casos refratários com surtos recorrentes de CTT. ${ }^{6}$ Assim, questionamos a

\section{Palavras-chave}

Síndrome de Takotsubo; Cardiomiopatia de Takotsubo; Recidiva; Revisão Sistemática.

Correspondência: Kenan Yalta •

Trakya University - Edirne 22030 - Turquia

E-mail: kyalta@gmail.com

Artigo recebido em 20/02/2020, revisado em 22/03/2020,

aceito em 22/03/2020

DOI: https://doi.org/10.36660/abc.20200140 incidência de $\mathrm{CMH}$ e doença cardíaca hipertensiva na análise geral. E em relação à reconstrução cirúrgica, se houver, e seu impacto na recorrência da CTT? Digno de nota, o maior risco de recorrência da CTT no sexo feminino ${ }^{1}$ pode, até certo ponto, ser atribuível à maior incidência de GMV sutil ou evidente, em sua maior parte associada a dimensões cavitárias relativamente pequenas e incidência relativamente maior de hipertrofia miocárdica significativa (em resposta à hipertensão sistêmica, etc.) em mulheres.

- A segunda categoria constitui a parte principal e pode incluir aqueles com uma descarga adrenérgica grave durante o evento índice de CTT. ${ }^{4,6,7}$ É importante ressaltar que a descarga adrenérgica grave é bem conhecida por estar significativamente associada a distúrbios inerentes ${ }^{6}$ que levam a mecanismos de resposta fisiológica exagerados (ao invés do grau do gatilho associado), potencialmente sugerindo sua natureza repetitiva e facilmente induzível em um determinado caso de CTT. A proximidade temporal com ao evento índice de CTT, sugerido como um fator de risco na análise atual, ${ }^{1}$ também pode substanciar o papel da descarga adrenérgica grave em futuras recorrências de CTT. Mais especificamente, a descarga adrenérgica grave, além de ser determinada diretamente com os níveis plasmáticos de catecolaminas, pode potencialmente apresentar uma variedade de sinais específicos, embora indiretos, incluindo gradiente agudo da via de saída do ventrículo esquerdo (VSVE) (na imagem cardíaca) e padrão de fluxo coronariano lento (FCL) (na angiografia coronária invasiva) em pacientes com CTT. ${ }^{4-7}$ Consequentemente, os casos com e sem recorrência de CTT diferiram significativamente em relação a esses sinais específicos durante seus eventos índice? Esses sinais ${ }^{4,6,7}$ também podem servir como potenciais preditores para recorrências de CTT? E quanto ao impacto das opções terapêuticas radicais, incluindo o bloqueio ${ }^{2,6,7}$ do gânglio simpático, se houver, nas recorrências de CTT?

Em suma, os autores ${ }^{1}$ devem ser parabenizados pela análise bem executada. Entretanto, mais estudos ainda são necessários para estabelecer completamente os preditores absolutos de recorrências futuras de CTT (possivelmente com a sugestão de um escore de risco simples), juntamente com estratégias de manejo específicas para a prevenção dessas recorrências. 


\section{Referências}

1. Campos FAD, Ritt LEF, Costa JPS, Cruz CM, Feitosa-Filho GS, Oliveira QB, et al. Factors Associated with Recurrence in Takotsubo Syndrome: A Systematic Review. Arq Bras Cardiol. 2020;114(3):477-83.

2. Yalta K, Yilmaztepe M, Zorkun C. Left Ventricular Dysfunction in the Setting of Takotsubo Cardiomyopathy: A Review of Clinical Patterns and Practical Implications. Card Fail Rev. 2018; 4(1): 14-20.

3. Azzarelli S, Galassi AR, Amico F, Giacoppo M, Argentino V, Fiscella A.. Intraventricular obstruction in a patient with tako-tsubo cardiomyopathy. Int J Cardiol. 2007; 121(2): e22-4.

4. Kawaji T, Shiomi H, Morimoto T,Tazaki J, Imai M, Sato N, et al. Clinical impact of left ventricular outflow tract obstruction in takotsubo cardiomyopathy. Circ J. 2015; 79(4): 839-46

\section{Carta-resposta}

Primeiramente, agradecemos aos autores os comentários a respeito de nosso recente artigo nos Arquivos Brasileiros de Cardiologia intitulado "Fatores Associados à Recorrência na Síndrome de Takotsubo: Uma Revisão Sistemática". ${ }^{1}$ $\mathrm{Na}$ carta, os autores fizeram alguns comentários adicionais interessantes sobre os mecanismos de recorrência de Takotsubo relacionados a gatilhos mecânicos associados ao gradiente médio-ventricular que podem estar relacionados à cardiomiopatia hipertrófica $(\mathrm{CMH})$ associada e a um segundo mecanismo relacionado à descarga adrenérgica grave.

Uma questão que eles colocaram estava relacionada às incidências de $\mathrm{CMH}$ e cardiopatia hipertensiva nos dados que analisamos. Na verdade, nos artigos originais que foram revisados, os autores não mencionaram as incidências de $\mathrm{CMH}$ ou cardiopatia hipertensiva. Alguns pacientes com Síndrome de Takotsubo (ST) podem apresentar movimento anterior sistólico da válvula mitral e obstrução ventricular, sendo que às vezes é difícil determinar se essas características estão relacionadas a ST ou $\mathrm{CMH}$ associada ou mesmo sua combinação e o acompanhamento clínico, eletrocardiográfico e de imagem pode revelar o diagnóstico. , $^{2,3}$ Sobre o impacto da reconstrução cirúrgica na recorrência, em uma breve revisão da literatura encontramos um artigo que descreve um caso de reconstrução ventricular em uma mulher que permaneceu com aneurisma

\section{Referências}

1. Campos FAD, Ritt LEF, Costa JPS, Cruz CM, Feitosa-Filho GS, Oliveira QB, et al. Factors Associated with Recurrence in Takotsubo Syndrome: A Systematic Review. Arq Bras Cardiol. 2020;114(3):477-83.

2. Sherid M<, Riedy K, Rosenzweig B, Massera D, Saric M, Swistel DG, et al. Distinctive Hypertrophic Cardiomyopathy Anatomy and Obstructive Physiology in Patients Admitted with Takotsubo Syndrome. Am J Cardiol. 2020; 125(11):1700-9

3. Vinardell JM, Mihos CG, Nader A, Ro R, Escolar E, Santana O. Stress Cardiomyopathy in a Patient with Hypertrophic Cardiomyopathy: Case Presentation and Review of the Literature. Rev Cardiovasc Med. 2018; 19(2): 65-8.

4. Kishida K, Woo E, Sasaki T, Hamori K, Daimon M, Mieno S, et al. Left Ventriculoplasty in a Patient With Suspected Takotsubo Cardiomyopathy Followed by a Left Ventricular Aneurysm. Kyobu Geka. 2014;67(5):419-22.
5. Yalta K, Yalta T. Physically triggered takotsubo cardiomyopathy has a worse prognosis: Potential roles of systemic inflammation and coronary slow flow phenomenon. Int J Cardiol. 2017;242: 31-32.

6. Yalta K, Yetkin E, Yalta T. Recurrent takotsubo cardiomyopathy: Further insights into morphological patterns. Cardiovasc Pathol. 2020; 48: 107225. doi:10.1016/j.carpath.2020.107225

7. Yalta K, Yalta T. Takotsubo cardiomyopathy and its implications in the setting of acute manic attack. Proc (Bayl Univ Med Cent). 2020;33(3):473-474. Published 2020 Jul 6. doi:10.1080/08998280.2020.1765664

apical e eventos embólicos recorrentes. ${ }^{4}$ Dessa forma, no caso de obstrução permanente da via de saída ventricular em pacientes com CMH que apresentavam ST pode-se considerar a indicação das diretrizes da CMH para intervenção cirúrgica. ${ }^{5}$

Em relação à descarga adrenérgica, concordamos que esta pode ser outra característica a ser lembrada em estudos futuros para melhor analisar sua relação com a gravidade e recorrência da ST. Estudos utilizando cintilografia miocárdica com 123I-MIBG demonstraram uma diminuição de captação na região com contratilidade ventricular comprometida e a persistência desse comprometimento estaria relacionada a maior chance de recorrência. ${ }^{6,7}$ Sobre o bloqueio ganglionar simpático, entendemos que pode ser um campo de pesquisa para pacientes com vários casos de recorrência; nenhum estudo foi encontrado em uma busca booleana no Pubmed com os termos bloqueio simpático ou bloqueio ganglionar ou bloqueio ganglionar cirúrgico e Takotsubo.

Novas pesquisas serão valiosas para uma melhor compreensão do prognóstico e das opções de tratamento para as síndromes de Takotsubo.

Luiz Eduardo Fonteles Ritt

Hospital Cardio Pulmonar - Centro de Estudos Clínicos Escola Bahiana de Medicina e Saúde Pública

5. Elliott PM, Anastasakis A, Borger MA,Borggrefe M, Cecchi F, Charron P, et al. 2014 ESC Guidelines on diagnosis and management of hypertrophic cardiomyopathy: the Task Force for the Diagnosis and Management of Hypertrophic Cardiomyopathy of the European Society of Cardiology (ESC). Eur Heart J. 2014;35(39):2733-79.

6. Verschure DO, Somsen GA, van Eck-Smit BL, Knol RJ, Booij J, Verberne HJ Tako-tsubo cardiomyopathy: how to understand possible pathophysiological mechanism and the role of (123)I-MIBG imaging. I Nucl Cardiol. $2014 ; 21(4): 730-8$.

7. Akashi YJ, Nakazawa K, Sakakibara M, Miyake F, Musha H, Sasaka K. 123I-MIBG myocardial scintigraphy in patients with "takotsubo" cardiomyopathy. J Nucl Med. 2004;45(7):1121-7.

Este é um artigo de acesso aberto distribuído sob os termos da licença de atribuição pelo Creative Commons 